\title{
Strength assessment of earth dams
}

\author{
Tokhirjon Sultanov ${ }^{1 *}$, Bakhtiyor Yuldoshev ${ }^{1}$, Elyor Toshmatov ${ }^{1}$, Javlonbek Yarashov ${ }^{1}$, \\ Rustam Ergashev ${ }^{1}$, and Mirziyod Mirsaidov ${ }^{1}$ \\ ${ }^{1}$ Tashkent Institute of Engineers of Irrigation and Mechanization in Agriculture, 100000 Qary- \\ Niyazov Str., 39, Tashkent, Uzbekistan
}

\begin{abstract}
A comparative method for estimating the stress-strain state of earth dams under the effect of static load is given in the paper using spatial and plane-strain models. The results of assessment of stress-strain state of several earth dams using these methods are given. Analysis of the results obtained allows us to conclude that a plane calculation model gives results almost identical to the ones obtained with spatial model; in future that can be the basis for developing recommendations on the use of certain calculation models. Some features of stress state in a spatial case are revealed, indicating the occurrence of dangerous areas with the greatest stresses.
\end{abstract}

\section{Introduction}

The reliability and safety of dams depends on stress-strain state (SSS) of a structure under various loads. Prediction of changes in stress-strain state of the dam allows obtaining complete information on structure strength.

Therefore, when assessing the strength, a reliable evaluation of the SSS of a structure is put in the forefront. Reliable determination of the SSS in turn depends on the chosen design scheme of the structure, mathematical models used for describing the processes, the equations of state of materials and methods for solving the problems under consideration [1-9].

Recently a number of papers have been published where both static and dynamic stressstrain state of various earth dams are considered in a plane statement [10-13].

The problem of spatial calculation of earth dams is a particularly laborious task [14-16]. Usually, when assessing the stress-strain state of dams located in wide gates, it is sufficient to use the conditions of plane strain; however, the use of plane calculation schemes for earth dams located in narrow gates requires more thorough verification of accuracy in assessing the SSS of structures.

The state of earth dam under various effects is also determined by its length. As noted in $[5,6]$ for extended dams (at the ratio of the crest length $L_{c r}$ to the height $H$ of the dam $L_{c r} / H \geq 6$ ), calculations can be made according to the plane strain scheme; in this case it is possible to consider in calculations not the whole structure, but only its central section. If the above ratio is violated, then the spatial nature of dam operation is manifested. In $[5,6$,

\footnotetext{
*Corresponding author: timiuz@mail.ru
} 
$14,15,16]$ it is shown that the accuracy of dam calculation changes not only under the action of static load but under dynamic effects as well.

The brief overview presented here shows that obtaining reliable results in calculating and estimating the SSS of earth dams is quite a serious task, since taking into account the real geometrical dimensions of earth dams dictates the necessity to consider the spatial character of structure operation to develop justified quantitative estimates of structure strength $[17,18]$.

Therefore, today it is necessary to develop the recommendations (criteria) for assessing the stress-strain state of earth dams using adequate plane or spatial calculation models that describe the real features of the structure.

\section{Methods of research}

This study is devoted to the solution of the following issues:

- to assess the stress-strain state of earth dams a plane model based on the plane-strain state of the structure and a spatial model that takes into account the geometric features of the structure are proposed;

- a variation problem is posed with allowance for the plane and spatial strain states of the structures under consideration;

- the methods for solving the tasks posed using plane and spatial finite elements under the action of static load are proposed;

- the stress-strain state of structures is investigated using plane and spatial models;

- it is necessary to draw primary conclusions on the use of plane or spatial models in assessing the SSS of the structures under consideration on the basis of analysis of the results obtained.

To estimate the SSS of earth dams, consider the spatial model of the structure shown in Fig.1; here the surfaces of the base and lateral slopes $\Sigma_{b}, \Sigma_{l_{1}}, \Sigma_{l_{2}}$ are rigidly fixed, the surface of the downstream face $\Sigma_{3}$ is stress-free, on the surface of $S_{p}$ hydrostatic pressure of water acts, and on the surface of the crest $\Sigma_{p}$ an external load $\Sigma_{2}$ is applied.

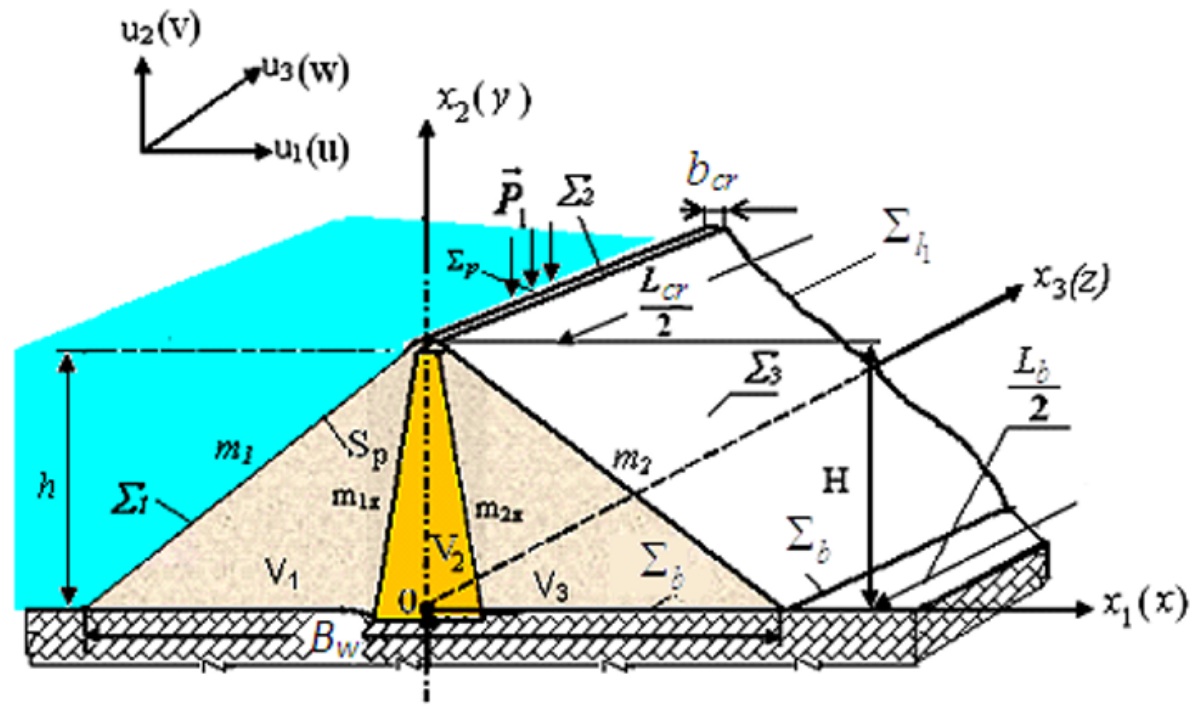

Fig.1. Spatial model of earth dams 
Here $V=V_{1}+V_{2}+V_{3}$ is the volume of the dam body $\left(V_{1}, V_{3}\right.$ - the volumes of the upper and lower thrust prisms, $V_{2}$ - the volume of the dam core); $\Sigma_{l_{1}}, \Sigma_{l_{2}}$ are the surfaces of the coastal slopes, $\Sigma_{b}$ is the surface of the base along the bottom, and $\Sigma_{1}, \Sigma_{3}$ и $\Sigma_{2}$ are the surfaces of thrust prisms and crest.

Material of the structure is considered to be elastic. The structure is taken as a massive structure, therefore, along with the above-listed loads, mass forces $\vec{f}$ are taken into account in calculations.

The task is to determine the fields of displacements and stresses in the elements of the structures $V=V_{1}+V_{2}+V_{3}$ under various static effects using two different models: plane and spatial ones.

To describe the spatial process of dam strain (Fig. 1) under static loads, the principle of possible displacements is used, according to which the sum of the work of all active forces acting on the structure at possible displacements is zero, that is

$$
\begin{gathered}
-\int_{V_{1}} \sigma_{i j} \delta \varepsilon_{i j} d V-\int_{V_{2}} \sigma_{i j} \delta \varepsilon_{i j} d V-\int_{V_{3}} \sigma_{i j} \delta \varepsilon_{i j} d V+ \\
\quad+\int_{V} \vec{f} \delta \vec{u} d V+\int_{S_{P}} \vec{p} \delta \vec{u} d S+\int_{\Sigma_{P}} \vec{P}_{1} \delta \vec{u} d \Sigma=0,
\end{gathered}
$$

Physical properties of the body material are described by the relationships between stresses $\sigma_{i j}$ and strains $\varepsilon_{i j}$ in the form [19]:

$$
\sigma_{i j}=\lambda_{n} \varepsilon_{k k} \delta_{i j}+2 \mu_{n} \varepsilon_{i j}
$$

connection between the components of strain tensor and displacement vector is described by the linear Cauchy relations [19]

$$
\varepsilon_{i j}=\frac{1}{2}\left(\frac{\partial u_{i}}{\partial x_{j}}+\frac{\partial u_{j}}{\partial x_{i}}\right)
$$

Homogeneous kinematic boundary conditions are used

$$
\begin{aligned}
& x \in \sum b: \vec{u}=0, \\
& i, j=1,2,3 \text {. }
\end{aligned}
$$

Here $\vec{u}, \varepsilon_{i j}, \sigma_{i j}$ are the displacement vector, strain and stress tensors, respectively; $\delta \vec{u}$, $\delta \varepsilon_{i j}$ - isochronous variations of displacements and strains; $\vec{f}$ is the vector of the mass forces; $\vec{P}_{1}$ - the vector of external forces; $\vec{p}$ - the hydrostatic water pressure applied to the site $\Sigma_{p}$ and $S_{p}$, respectively; index $n=1,2,3$ denotes the part of the structure to which this mechanical characteristic relates.

In calculations, different levels of reservoir filling are considered, so, the ordinates of the hydrostatic water pressure on the upstream face of the dam are determined by the formula

$$
\vec{p}=\rho_{\mathrm{O}} g(h-y) .
$$


Now the problem under consideration can be formulated as follows: it is necessary to determine the fields of displacement $\vec{u}(\vec{x})$ and stresses $\sigma_{i j}(\vec{x})$ in the body of the dam $\mathrm{V}$ under the action of $\vec{f}, \vec{P}_{1}$ and $\vec{p}$, satisfying equations (1), (2), (3) and kinematic conditions (4) on any possible displacement $\partial \vec{u}$.

When assessing the SSS of the dam using the model of plane strain, the indices $i, j$ in the ratio (1)-(3) take the value $i, j=1,2$ and the integration is performed over the surface of central section of the dam; the problem is simplified by an order of magnitude.

Further, the above-stated variation problem (1)-(4) of assessment the SSS of earth dams (Fig.1) under hydrostatic pressure and mass forces, taking into account spatial factors, with the use of a finite element in the form of a hexagonal parallelepiped having 24 degrees of freedom is reduced to a resolving system of algebraic equations of the $N$-th order:

$$
[K]\{u\}=\{P\}
$$

where the elements of the stiffness matrix $[K]$ of the structure (Fig.1) are constant and depend on elastic physical-mechanical parameters of the structure; $\{u\}$ - the sought for vector of nodal displacements; $\{P\}$ - the vector of external load (mass forces and hydrostatic water pressure).

When the equation (6) is formed, the stiffness matrices $[K]$ and nodal forces $\{P\}$ are formed automatically using the algorithm given in [4, 20].

Kinematic boundary conditions (4) are taken into account in the formation of the system of equations (6), restricting its order to equations that do not contain zero displacements. The order of the generated systems of algebraic equations (6) in some calculations exceeds 2000 .

When considering this problem in a plane statement, the triangular finite element is used to discretize the structure and the problem is reduced to an equation of the form (6).

In both cases, the solution of the obtained systems (6) is carried out by the Gauss method or the square root method [21,22], considering the ribbon structure of the stiffness matrix. Solving the system of equations (6), the displacement components $\left(u_{1}, u_{2}, u_{3}\right)$ are determined at each point of the structure (that is, the fields of displacements), then using these data the components of the tensors of strains (3) and stresses (2) are determined taking into account the inhomogeneous constructive features of dams.

\section{Results of research}

Using plane and spatial models of earth dam and the developed methods, the stress-strain state of the structure is determined in the central section and near the coastal slopes; this state occurs under the action of gravitational forces (own weight) and hydrostatic water pressure, taking into account the homogeneous and inhomogeneous scheme of the structure and elastic properties of material.

Above results for the dams considered are obtained taking into account their inhomogeneous and constructive features.

In concrete calculations, the order of the resolving system of equations (6) for a plane model reached 2000, for a spatial model -6000 .

Figures 2 and 3 show the isolines of distribution of stress tensor components for the central section of the Gissarak (Fig. 2) and Sokh (Fig. 3) dams under their own weight, obtained using plane and spatial models of structures. In the above figures, the left column shows a spatial calculation and the right one - plane calculation. The stresses in all figures are given in $\mathrm{MPa}$. 
Coordinate axes in Fig. 1 have the following directions: $x_{1}-$ (horizontal) and $x_{2}-$ (vertical) are in the plane of the central section; $x_{3}$ is perpendicular to this plane.

Comparison of the results for spatial (Fig. 2 - left) and plane models (Fig.3 - right) for the Gissarak dam shows the same pattern of stress distribution and their close values, i.e. when a vertical gravity load is applied to the dam, the pattern of the distribution of stress components $\left(\sigma_{11}, \sigma_{12}, \sigma_{22}\right)$ in the central section of the dam is identical. The maximum values of both horizontal $\sigma_{11}$ and vertical $\sigma_{22}$ stresses are reached at the bottom of the central - the highest - part of the dam, and tangential stresses $\sigma_{12}$ along the slopes. Stress distribution (Fig.2) with respect to the $\mathrm{x} 3$ axis is almost symmetrical, since the structure itself is almost symmetrical, with a slight difference in the slope inclinations $(\mathrm{m} 1=2.2$, $\mathrm{m} 2=1,9)$.
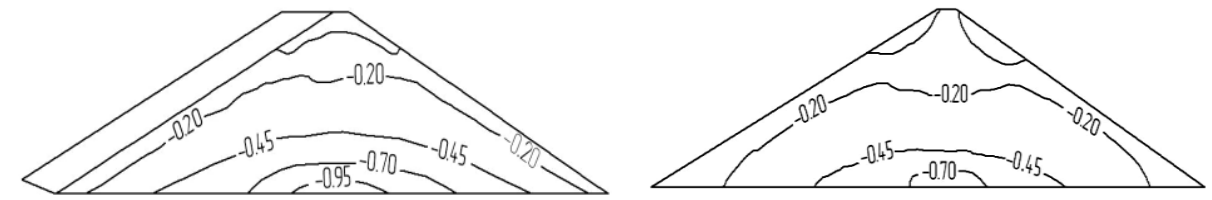

Normal horizontal stresses $-\sigma_{11}$
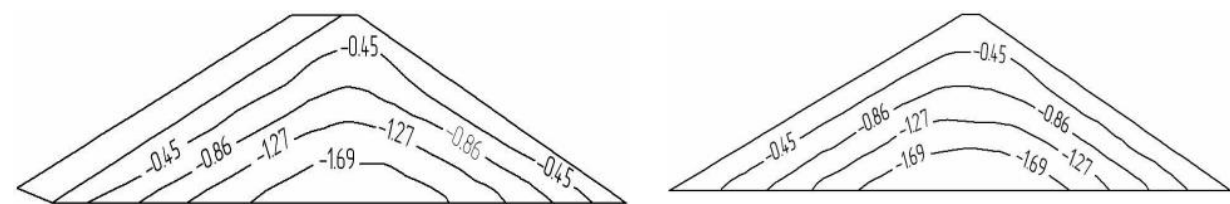

Normal vertical stresses $-\sigma_{22}$
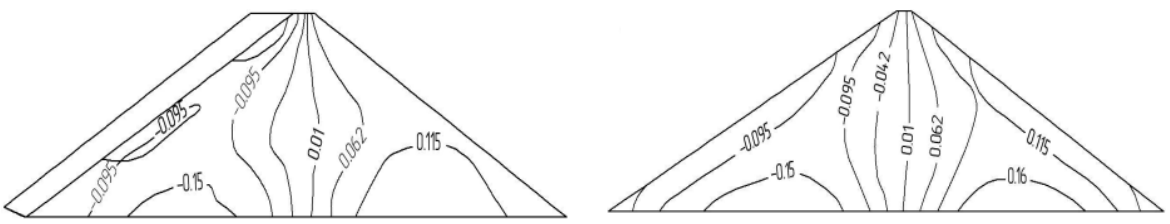

Tangential stresses $-\sigma_{12}$

Fig.2. Isolines of the distribution of stress tensor components in the central section of the Gissarak dam under own weight for spatial (left) and plane (right) models

The condition of rigid fixing is set near the coastal zones of the structure contacting with the mountain slopes. Here the results obtained with spatial model do not have plane analogs. When calculating this zone a spatial model should be used.

For this dam, the ratio of the geometric dimensions (the length of the crest to the height $-L_{c r} / H$, the length of the base to the height $-L_{b} / H$ and the width of the base to the height $\left.B_{w} / H\right)$ are $L_{c r} / H=4.76, L_{b} / H=1.43, B_{w} / H=4.57$, respectively. Therefore, the use of plane and spatial models for the central zone gives almost identical results, which means that in static calculations of such dams the use of plane models is possible.

Comparison of the isolines of distribution of stress components for the Sokh dam (Fig.3) using plane (Fig.3 - right) and spatial (Fig.3 - left) models shows a significant difference in the results obtained. Analysis of the spatial stress state and the maximum values of normal horizontal $\sigma_{11}$ and vertical stresses $\sigma_{22}$ at the bottom of central - the highest - part of the dam, and the tangential stresses $\sigma_{12}$ along the slopes and in the upper 
thrust prism indicate that the calculation of this dam based on a plane model does not provide the necessary accuracy.
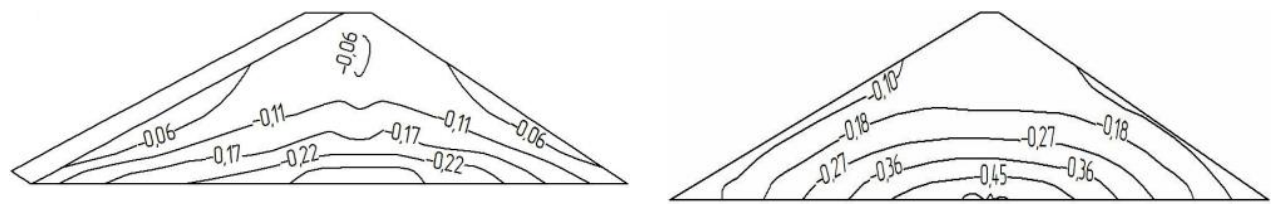

Normal horizontal stresses - $\sigma_{11}$
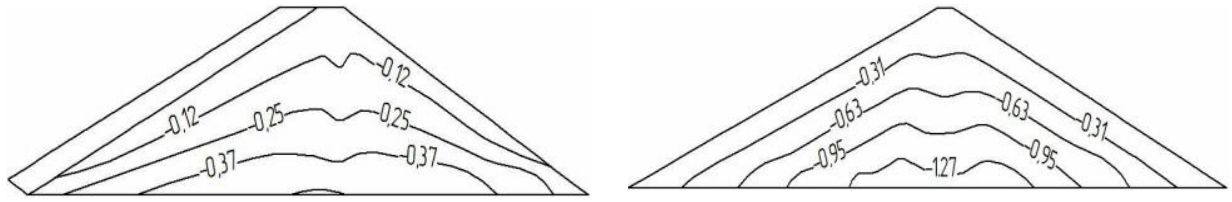

Normal vertical stresses $-\sigma_{22}$

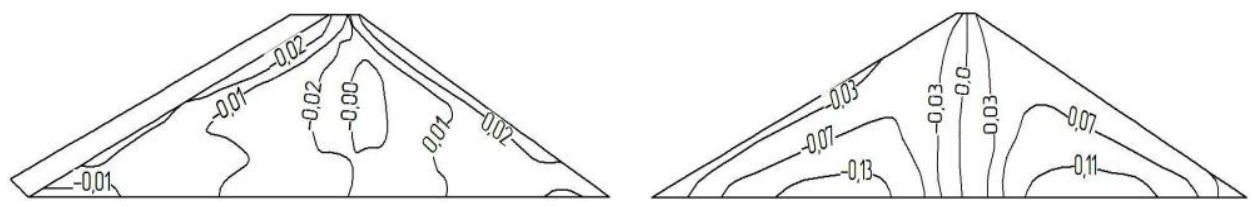

Tangential stresses $-\sigma_{12}$

Fig.3. Isolines of the distribution of stress tensor components in the central section of the Sokh dam under own weight for spatial (left) and plane (right) models

This is apparently explained by the small ratio of the horizontal dimensions of the dam to its height: $L_{c r} / H=0.56, L_{b} / H=0.24, B_{w} / H=6.07$. In this case, the structure is not long and does not correspond to the condition for choosing a plane calculation model. It must be regarded as a spatial body.

Similar verification calculations of the ratio of horizontal dimensions to the height have been made for other dams: the Akhangaran $\left(L_{c r} / H=19.3, L_{b} / H=7.21, B_{w} / H=5.2\right)$, Zaamin $\left(L_{c r} / H=5.55, L_{b} / H=2.15, B_{w} / H=5.76\right)$, Pachkamar $\left(L_{c r} / H=8.18, L_{b} / H=8.19, B_{w} / H=9.06\right)$, Nurek $\left(L_{c r} / H=2.46, L_{b} / H=1.01, B_{w} / H=4.79\right)$.

Results obtained for these dams with spatial and plane models showed that the maximum difference in the values of the stress tensor components is $7-9 \%$, in most cases the calculation results are identical.

In complex calculations (for the Hissarak, Akhangaran, Zaamin, Pachkamar and Nurek dams), this fact gives grounds for using a plane calculation model under gravitational load. Analysis of the stressed state of central section of the Gissarak Dam under its own weight, for two models considered (Fig.2) shows the qualitative and quantitative identity of stresses across the cross section.

Thus, the stress state of the central cross section of dams, geometrically similar to the Gissarak dam, can be described with satisfactory accuracy by a plane model.

As for the stresses in the gate plane, they could be calculated on the basis of a spatial model. 
An analysis of the stress state of the dams examined shows that the maximum tangential stresses $\sigma_{23}$ at vertical gravitational load arise along the lateral boundaries of the gates and can cause shear and cracks in the sides.

Vertical normal stresses $\sigma_{22}$ occur in the central part of the dam base. The maximum (in the modulus) horizontal stresses $\sigma_{11}$ appear at the base along the crest; their negative value in the central part indicates the compression of the central part under the crest, and the positive values - the heaving of the lower part of the side slopes when the dam body is settled under its own weight (Fig.3).

Positive values of horizontal stresses $\sigma_{33}$ in the upper part of the sides indicate the possibility of cracks and ruptures in lateral slopes. Thus, a spatial model makes it possible to evaluate the stress-strain state not only in the cross section, but also in dangerous zones in the points where the dam connects to the gorge. The comprehensive analysis of the stressed state of the dams under gravitational forces has shown that to estimate the SSS of cross-section of all the dams considered (with the exception of the Sokh dam) it is possible to use a plane model, and near the end sections, where a spatial stress state is manifested to use a spatial model. To assess the SSS of Sokh dam and other dams similar in geometry a spatial model should be used. Analysis of the stress state of various dams under gravitational loads has shown that two models developed with account of geometric features of structures, truly describe the processes occurring in earth dams. The possibility of their use in solving specific problems should be justified by the geometric dimensions and design features of the structures in question.

\section{Conclusions}

1. Methods for assessing the stress-strain state of earth dams under static load are presented using a spatial and plane-strain model.

2. The stress-strain state of various earth dams is estimated with the use of plane and spatial models.

3. The conclusion is drawn in what cases it is possible to use plane-strain models to assess the stress-strain state of earth dams, which give almost identical results with spatial ones.

4. Spatial calculation has made it possible to identify dangerous sections of the structure, where high stresses occur to compare with other sites. Captions should be typed in 9-point Times. They should be centred above the tables and flush left beneath the figures.

\section{References}

1. V.B. Glagovsky, O.M. Finagenov, Izvestiya VNIIG B.E. Vedeneev, 238 (2000)

2. Yu.K. Zaretsky, V.N. Lombardo, Static and dynamics of earth dams (Moscow, Energoizdat, 1983)

3. N.D. Krasnikov, Seismic stability of hydro-technical structures erected form earth materials (Moscow, Energoizdat, 1981)

4. M.M. Mirsaidov, Theory and methods for calculating earth structures for strength and seismic resistance (Tashkent, Fan, 2010)

5. Ya.I. Natarius, Hydrotechnical construction, 10 (1978)

6. Ya.I. Natarius, Increase in seismic resistance of dams erected from earth materials (Moscow, Energoatomizdat, 1984)

7. R. Afiri, S. Gabi, Infrastruct. Solut, 3 (2018) 
8. T.Wang, J.-sh. Chen, T. Wang, Sh.Wang, Natural Hazards, 76, 2 (2015)

9. V.S. Prokopovich, A.S. Velichko, R.N. Orishchuk, Izvestiya of the All-Russian Scientific Research Institute of Hydraulic Engineering named after. B.E. Vedeneev, 282 (2016)

10. M.M. Mirsaidov, T.Z. Sultanov, R.A. Abdikarimov, A.N. Ishmatov, B.Sh. Yuldoshev, E.S. Toshmatov, D.P. Jurayev, Magazine of Civil Engineering, 1 (2018)

11. M.M. Mirsaidov, T.Z. Sultanov, D.A. Khodzhaev, Civil-Comp Press, Stirlingshire, 108 (2012)

12. M.M. Mirsaidov, T.Z. Sultanov, Magazine of Civil Engineering, 5 (2014)

13. T.Z. Sultanov, D.A. Khodzhaev, M.M. Mirsaidov, Magazine of Civil Engineering, 1 (2014)

14. A.M. Abarin, PhD Thesis, Moscow (1999)

15. M.M. Mirsaidov, T.Z. Sultanov Scientific papers of the Republican Scientific and technical Conference with the Participation of Foreign Scientists "Resource-saving technologies in railway transport" December 15-16 (2011)

16. L.N. Rasskazov, A.S. Bestuzheva, P.L. Nguen. Seismic Stability of Earth Dams in Spatial Statement (2010)

17. M.M. Mirsaidov, 6th International Conference on Dam Engineering, 15-17 February (2011)

18. T.Z. Sultanov, M.M. Mirsaidov, D.A. Khodjaev Proc. of Australasian Structural Engineering Conference, 11-13 July (2012)

19. M.A. Koltunov, A.S. Kravchuk, V.P. Mayboroda, Applied mechanics of the deformed rigid body (Moscow, High school, 1983)

20. K. Bate, E. Wilson, Numerical methods of analysis and FEM (Moscow, Stroiizdat, 1982)

21. I.S. Berezin, N.P. Zhidkov, Methods of computation (Moscow, Science, 1969)

22. D.K. Fadeev, V.N. Fadeeva, Computational methods in linear algebra (Moscow, Fizmatgiz, 1960) 